\title{
A eficácia da fisioterapia na ansiedade em indivíduos com fibromialgia: revisão sistemática
}

\author{
Efficacy of physiotherapy in anxiety in fibromyalgia individuals: systematic review
}

La eficacia de la fisioterapia en la ansiedad en personas con fibromyalgia: una revisión sistemática

Samira Veras Cunha ${ }^{1}$, Rebeca Galdino Medeiros ${ }^{1}$, Rosangela Lago da Silva ${ }^{1}$, Guilherme Pertinni de Morais Gouveia ${ }^{1,2 *}$.

\section{RESUMO}

Objetivo: Analisar a eficácia da Fisioterapia na ansiedade de indivíduos com fibromialgia, por meio de uma revisão sistemática. Métodos: Revisão sistemática, a partir do fluxograma PRISMA, incluindo estudos quantitativos primários experimentais, artigos de livre acesso, disponíveis em português, inglês e espanhol, publicados entre 2015 e 2019, utilizando a estratégia PICOS por meio do site parsif.al®. Foram encontrados 168 artigos durante os meses de junho e julho de 2019, mediante as bases Lilacs, Scielo, Pubmed, MedLine, PeDro, Cochrane, Science direct e Google acadêmico. Para avaliar a qualidade dos ensaios clínicos randomizados, utilizou-se a Escala de Jadad. Resultados: Foram incluídos 24 estudos, dos quais 15 compuseram a amostra final. As pesquisas trazem que a partir da diminuição dos sintomas físicos nos pacientes com fibromialgia, as complicações mentais, como os níveis de ansiedade, sofrem reduções. Considerações finais: Esta revisão identificou que a combinação de diferentes intervenções fornecidas individualmente ou em grupo a pacientes adultos com fibromialgia exerce influência na redução de complicações sintomatológicas, melhorando os níveis de ansiedade.

Palavras-chave: Fisioterapia, Fibromialgia, Ansiedade.

\begin{abstract}
Objective: to analyze the effectiveness of physical therapy in the anxiety of individuals with fibromyalgia, through a systematic review. Methods: Systematic review, based on the PRISMA flowchart, including primary quantitative studies, free access articles, available in Portuguese, English and Spanish, published between 2015 and 2019, using a PICOS strategy through the website parsif.al $\AA^{\text {. }} 168$ articles were found during the months of June and July 2019, using as bases Lilacs, Scielo, Pubmed, Medmed, MedLine, PeDro, Cochrane, Science direct and Google scholar. To assess the quality of randomized controlled trials, use the Jadad Scale. Results: 24 studies were included, of which 15 were completed. Research shows that from the physical symptoms in patients with fibromyalgia, such as mental complications, such as anxiety levels, stress reductions. Final considerations: This review identified that the combination of different types of use used or in the group of adult patients with fibromyalgia influences the reduction of symptomatic complications, improving levels of anxiety.
\end{abstract}

keywords: Physiotherapy, Fibromyalgia, Anxiety.

1 Universidade Federal do Delta do Parnaíba (UFDPar), Parnaíba - PI.

2 Universidade Federal do Ceará (UFC). Fortaleza - CE *E-mail: gpfatufpi@gmail.com 
RESUMEN

Objetivo: analizar la efectividad de la fisioterapia en la ansiedad de las personas con fibromialgia, a través de una revisión sistemática. Métodos: Revisión sistemática, basada en el diagrama de flujo PRISMA, que incluye estudios cuantitativos primarios, artículos de acceso libre, disponibles en portugués, inglés y español, publicados entre 2015 y 2019, utilizando una estrategia PICOS a través del sitio web parsif.al®. Se encontraron 168 artículos durante los meses de junio y julio de 2019, utilizando Lilacs, Scielo, Pubmed, Medmed, MedLine, PeDro, Cochrane, Science direct y Google Scholar como bases. Para evaluar la calidad de los ensayos controlados aleatorios, use la Escala de Jadad. Resultados: se incluyeron 24 estudios, de los cuales 15 se completaron. La investigación muestra que a partir de los síntomas físicos en pacientes con fibromialgia, como complicaciones mentales, como niveles de ansiedad, reducción del estrés. Consideraciones finales: Esta revisión identificó que la combinación de diferentes tipos de uso utilizados o en el grupo de pacientes adultos con fibromialgia influye en la reducción de complicaciones sintomáticas, mejorando los niveles de ansiedad.

Palabras clave: Fisioterapia, Fibromialgia, Ansiedad.

\section{INTRODUÇÃO}

A fibromialgia (FM) é uma síndrome crônica e dolorosa e, por essa razão, acaba por comprometer a qualidade de vida da pessoa, podendo dificultar as suas atividades cotidianas (MARTINS RCC e SILVEIRA NC, 2018). Sua prevalência na população em geral gira em torno de 0,2 e $6,6 \%$, sendo que na população feminina é ainda maior, variando entre $2,4 \%$ e 6,8\% (MARQUES AP, et al., 2017).

Tal condição engloba aspectos clínicos como a dor crônica e generalizada, acompanhada de fadiga, alterações no sono, dificuldades de memória, alterações no humor, sintomas somáticos e outros (WOLFE F, 2015). Indivíduos com fibromialgia apresentam diversos transtornos mentais como depressão, ansiedade, mal-estar físico, baixa alta estima e alteração de humor, comprometendo-os em sua qualidade de vida e desempenho diário (GONDIM SS e ALMEIDA MAPT, 2018).

Um estudo realizado com 140 participantes promoveu uma estatística sobre a qualidade de vida desses indivíduos, no qual $50 \%$ dos inquiridos percepcionaram a doença como incapacitante, enquanto $45,7 \%$ a vê como moderadamente incapacitante. Paralelamente, no mesmo estudo, os resultados do questionário de impacto da fibromialgia, corresponderam a uma pontuação média de 63,76 pontos, indicando má qualidade de vida (MARTINS R, et al., 2016).

A ansiedade é uma experiência emocional em função da previsão de situações futuras ou em presença de circunstâncias consideradas como desagradáveis para o indivíduo. Essa se refere a um momento transitório, que pode ser caracterizado por apreensão, por tensão e por elevação das atividades do sistema nervoso autônomo (CHAVES ECL, et al., 2015). Quando uma pessoa é resignada a uma situação a qual foi desagradável, resulta em um alto grau de ansiedade (GUIMARÃES AMV, et al., 2015).

Nos últimos quatro anos, o número de pesquisas relacionadas à eficácia de intervenções fisioterapêuticas sobre a ansiedade, cresceram. Estudos apontam que a promoção de técnicas para relaxamento muscular progressivo, ajuda a evitar espasmos musculares e no controle da tensão auxiliando na diminuição da dor e rebaixando a ansiedade colaborando assim para a qualidade de sono e distração (RODRIGUES GF, et al., 2017).

Nesse contexto, questiona-se: qual a eficácia das intervenções fisioterapêuticas na redução dos níveis de ansiedade em indivíduos com fibromialgia? Portanto, os resultados dessa pesquisa auxiliarão os profissionais de saúde na escolha de um método mais adequado, propiciando assim, o desenvolvimento do melhor tratamento, proporcionando positivos desfechos clínicos dos pacientes. Portanto, objetivou-se com este estudo analisar a eficácia da Fisioterapia na ansiedade de indivíduos com fibromialgia, por meio de uma revisão sistemática. 


\section{MÉTODOS}

\section{Desenho de estudo}

Trata-se de uma revisão sistemática, a partir do fluxograma do "Preferred Reporting Items for Systematic Reviews and Meta-Analyses" (PRISMA), incluindo estudos quantitativos primários experimentais ou quase experimentais. As intervenções dos ensaios clínicos randomizados foram avaliadas de acordo com a eficácia.

\section{Critérios de inclusão}

Artigos de livre acesso, disponíveis em português, inglês e espanhol, publicados de 2015 a 2019. Esse período foi determinado, devido ao aumento das pesquisas que mostram relação do tratamento fisioterápico com os sintomas de ansiedade na fibromialgia. Foram pesquisados 168 artigos durante os meses de junho e julho de 2019, através das bases de dados Lilacs, Scielo, Pubmed, MedLine, PeDro, Cochrane, Science direct e Google acadêmico, evitando possível viés de publicação. Os artigos contêm informações essenciais aos profissionais de saúde sobre tal assunto, de modo que a aplicação deste conhecimento e a aquisição de habilidades melhorem o cuidado e promovam o alcance de desfechos positivos nos indivíduos com ansiedade.

\section{Protocolo do estudo}

\section{Estratégia de busca}

A estratégia PICOS (P - population; I - intervention; C - comparison; O - outcomes e S - study) guiou a elaboração da pergunta norteadora da RS e serviu de base para o desenvolvimento das estratégias de busca utilizando-se os Descritores em Ciências da Saúde (DECS), com os operadores booleanos OR e AND, conforme apresentado no Quadro 1. Foi utilizado para a sua execução o site parsif.al®. Ressalta-se que o elemento $C$ da estratégia PICO não foi abordado, pois não se trata de um estudo comparativo. Assim, a questão de pesquisa delimitada foi: "qual a eficácia das intervenções fisioterapêuticas na redução dos níveis de ansiedade em indivíduos com fibromialgia? " Nela, o primeiro elemento da estratégia (P) consiste no indivíduo com fibromialgia apresentando sintomas de ansiedade; o segundo (I), as intervenções fisioterapêuticas; e o quarto elemento $(\mathrm{O})$ a eficácia da fisioterapia sobre a ansiedade nesses pacientes; como (S) foram considerados para inclusão todos os tipos de estudos a partir de 2015. As buscas foram realizadas utilizando-se as estratégias do Quadro 2.

Quadro 1 - Elementos da estratégia PICO, descritores e palavras-chave, Piauí, Brasil, 2019.

\begin{tabular}{|l|l|l|l|}
\hline Componente & Definição & Descritores & Palavras-chave \\
\hline $\begin{array}{l}\text { P: população } \\
\text { de interesse }\end{array}$ & $\begin{array}{l}\text { Indivíduos com } \\
\text { fibromialgia com } \\
\text { sintomas de ansiedade }\end{array}$ & $\begin{array}{l}\text { Não foram adotados descritores ou palavras-chave voltados } \\
\text { para a população nessa pesquisa }\end{array}$ \\
\hline I: intervenção & $\begin{array}{l}\text { Intervenções } \\
\text { fisioterapêuticas }\end{array}$ & $\begin{array}{l}\text { Fibromialgia } \\
\text { Ansiedade } \\
\text { Anxiety } \\
\text { Qualidade de vida } \\
\text { Quality of life }\end{array}$ & $\begin{array}{l}\text { Complicações da fibromialgia } \\
\text { Sintomas na fibromialgia }\end{array}$ \\
\hline $\begin{array}{l}\text { C: } \\
\text { comparação }\end{array}$ & - & - & - \\
\hline $\begin{array}{l}\text { O: resultado/ } \\
\text { desfecho }\end{array}$ & $\begin{array}{l}\text { Eficácia da fisioterapia } \\
\text { sobre a ansiedade } \\
\text { nesses pacientes }\end{array}$ & $\begin{array}{l}\text { Modalidades de fisioterapia } \\
\text { Physical } \\
\text { Modalities }\end{array}$ & $\begin{array}{l}\text { Tratamentos fisioterápicos na } \\
\text { fibromialgia } \\
\text { Influência da fisioterapia na } \\
\text { fibromialgia } \\
\text { A fisioterapia na ansiedade }\end{array}$ \\
\hline
\end{tabular}

Fonte: Cunha SV, et al., 2020. 
Quadro 2 - Estratégias de busca utilizadas nas bases de dados, Piauí, Brasil, 2015 - 2019.

\begin{tabular}{|c|c|}
\hline $\begin{array}{c}\text { Base de dados } \\
\text { Biblioteca online }\end{array}$ & Estratégias de busca \\
\hline LILACS & $\begin{array}{r}\text { Filtros: idioma (inglês, espanhol e português) Ano de publicação: (2015 - 2019) } \\
\text { e tipo de documento (artigo). }\end{array}$ \\
\hline Medline & $\begin{array}{c}\text { (Physiotherapy) AND (Fibromyalgia) OR (Anxiety) } \\
\text { Filtros: idioma (inglês, espanhol e português) }\end{array}$ \\
\hline SciELO (via scielo.br) & Ano de publicação: (2015 - 2019) e tipo de documento (artigo) \\
\hline PEDro & $\begin{array}{c}\text { (Physiotherapy) AND (Fibromyalgia) AND (Anxiety) } \\
\text { (inglês, espanhol e português) Ano de publicação: (2015 - 2019) }\end{array}$ \\
\hline Pubmed & $\begin{array}{c}\text { (Physiotherapy) AND (Fibromyalgia) AND (Anxiety) } \\
\text { Ano de publicação: (2015 - 2019) }\end{array}$ \\
\hline Science direct & $\begin{array}{c}\text { (Physiotherapy) AND (Fibromyalgia) AND (Anxiety) } \\
\text { Ano de publicação: (2015 - 2019) }\end{array}$ \\
\hline Cochrane & (Physiotherapy) AND (Fibromyalgia) AND (Anxiety) \\
Ano de publicação: (2015 - 2019)
\end{tabular}

Fonte: Cunha SV, et al., 2020.

\section{Seleção dos artigos}

Inicialmente foi realizada a análise de títulos, seguida da leitura dos resumos para a identificação daqueles que seriam avaliados na integra, de forma independente, por quatro pesquisadores. Extraíram-se os dados necessários finais por meio de um instrumento contendo dados de identificação (autores e ano), desenho do estudo, tipo de população, tipos de técnicas fisioterapêuticas e eficácia sobre a ansiedade desses pacientes.

\section{Avaliação da qualidade metodológica dos estudos}

Para avaliar a qualidade dos ensaios clínicos randomizados, utilizou-se a Escala de Jadad, que consiste em três itens diretamente relacionados a redução de viés da pesquisa (randomização, cegamento e destino de todos os participantes), totalizando 5 pontos. Os estudos são classificados como de má qualidade se a pontuação for inferior a 3. Tais itens com pontuação baixa foram excluídos.

\section{Análise dos resultados}

Os dados dos estudos incluídos nessa revisão foram classificados a partir de seus desfechos/complicações e apresentados de forma descritiva. Não foi possível realizar metanálise, devido a diversidade das intervenções e amostras dos estudos.

\section{RESULTADOS}

Com base nas estratégias de busca e na seleção apresentadas na seção dos métodos, foram incluídos 24 estudos para leitura na integra, dos quais 15 compuseram a amostra final. Processo de seleção desses estudos (Figura 1). 
Figura 1 - Fluxograma do processo de seleção dos estudos segundo o PRISMA, Piauí, Brasil, 2019.

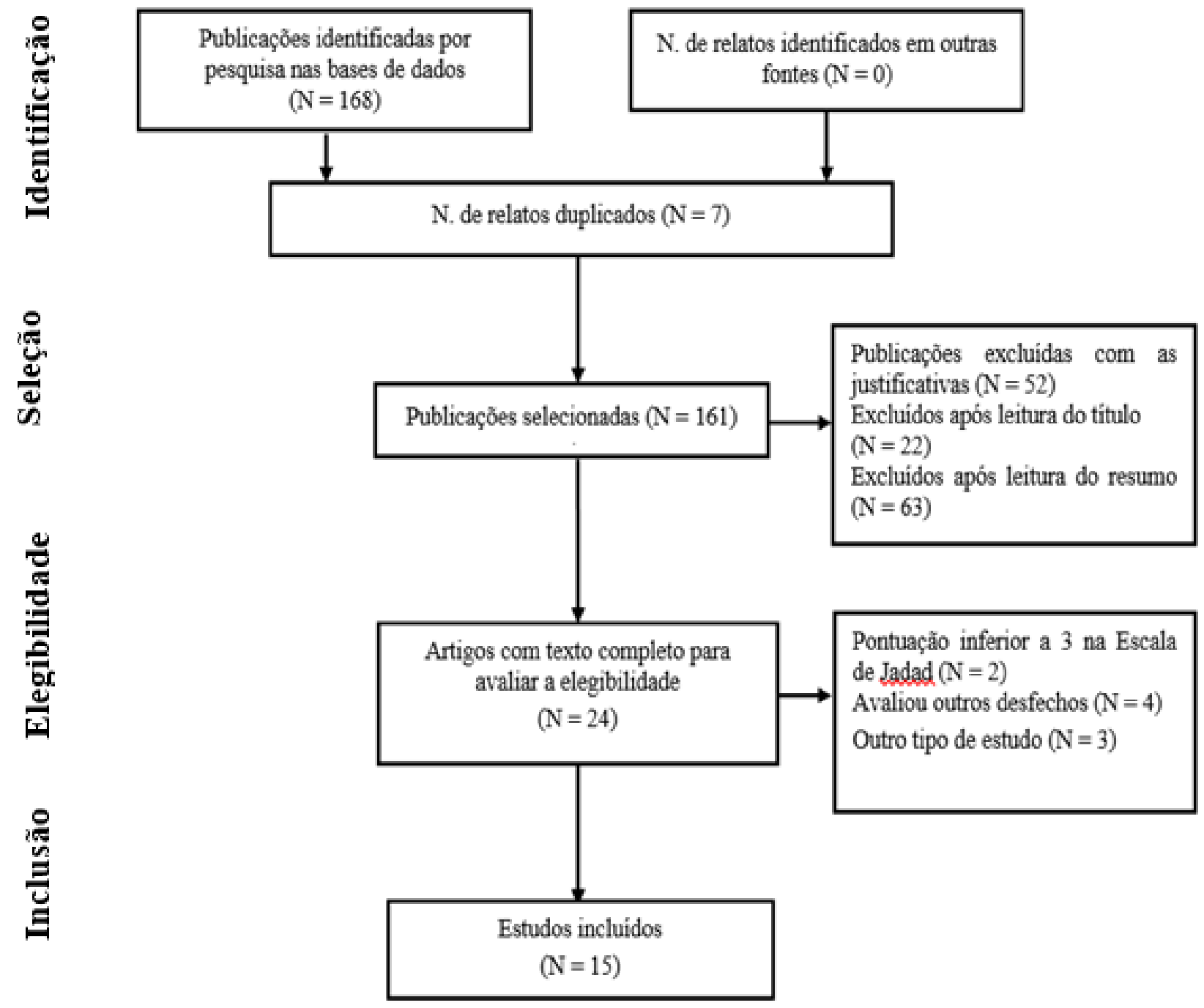

Fonte: Cunha SV, et al., 2020.

\section{Características gerais e qualidade dos estudos}

O Quadro 3 apresenta as principais características, os resultados e a qualidade dos ensaios clínicos randomizados $(n=15)$. Os estudos foram realizados em diferentes países: Brasil $(n=3)$, Estados Unidos $(n=$ 1), Espanha $(n=5)$, Suécia $(n=1)$, Bélgica $(n=1)$, Turquia $(n=2)$, Itália $(n=1)$ e Austrália $(n=1)$. As características das amostras eram distintas: o número de pacientes avaliados variou de 15 a 226, e a idade média de 18 a 60 anos. Os estudos tiveram em sua maioria, participantes do sexo feminino. O tempo médio de resultados positivos quanto aos sintomas da FM nos estudos, é de 3 semanas, quando mostram estatísticas significativas. $O$ tempo de acompanhamento dos pacientes após a intervenção variou de 3 meses a 1 anos.

\section{Profissionais que implementaram as intervenções}

Todos os estudos apresentados nos resultados possuem intervenções fisioterápicas. Entretanto, alguns estudos possuem intervenções multiprofissionais, como a de psicólogos, educadores físicos e médicos para prescrição de fármacos (grupo controle).

\section{Efetividade e eficácia das intervenções}

Dos 15 estudos identificados, dois estudos mostraram eficácia da intervenção a partir de terapia comportamental, para redução de estresse, ansiedade, dor e melhora na qualidade de movimento (BONGI 
SM, et al., 2016; ROCHA MO, et al., 2017). Outro estudo também utilizou a técnica de terapia comportamental, porém, adicionada a outras intervenções, como técnicas fisioterápicas para capacidade aeróbica, fortalecimento muscular, flexibilidade, atendimentos de hidrocinesioterapia e cinesioterapia (BRAVO C, et al., 2017), proporcionando melhoras com relação à dor, distúrbios psicológicos, funcionalidade, sono e qualidade de vida. A terapia comportamental foi utilizada ainda, juntamente com exercícios físicos e técnica de mobilização articular cervical superior (GUIMARÃES AMV, et al., 2015), proporcionando eficácia quanto a melhora da dor, fadiga muscular, depressão, ansiedade e assim, qualidade de vida.

A pompage associada a exercícios de alongamento e exercícios aeróbicos durante doze semanas para tratamento da FM não apresentou efeitos benéficos, visto que não influenciou sobre o limiar de ansiedade, fadiga muscular e depressão, mostrando apenas uma pequena eficácia em um dos testes de dor no estudo (GOES SM, et al., 2017). A crioterapia juntamente a fisioterapia, mostrou efeitos positivos rápidos com relação aos estados físico e mental dos indivíduos (MARTINS RCC e SILVEIRA NC, 2018). Técnicas de Tai chi mostraram efetividade nos níveis de depressão, ansiedade e outros comportamentos mentais (MARTINS R, et al., 2016).

Em um estudo comparativo entre técnicas de Pilates e massagem tecidual, houve maior eficácia quanto a ansiedade dos participantes, a partir dos exercícios de Pilates (SILVA HJA, et al., 2019). Já em um estudo comparando terapia a partir de Dry Needling (agulhamento a seco) com técnica de liberação miofascial, relatou que ocorreu uma melhora, mais eficaz para sono, depressão, ansiedade, cansaço e intensidade da dor, nos indivíduos que passaram pela terapia com Dry Needling (CASTEL A, et al., 2014).

Exercícios respiratórios, posturais e técnicas de Tai Ji Quan mostraram melhora na qualidade de vida e ansiedade (ANDRADE GS et al., 2018). Em outra pesquisa, usando terapia combinada com fototerapia e treinamento físico, proporcionou eficácia quanto aos níveis de ansiedade, depressão e fadiga, porém não mostrou diferença significativa comparada ao fármaco com relação ao distúrbio de sono (CHAVES ECL, et al., 2015). Técnicas de sofrologia (FERNANDO RS, et al., 2017), mostraram relevantes melhoras físicas e mentais.

Uma pesquisa utilizou técnicas de mobilização neurodinâmica, na qual o tratamento reduziu a sensibilidade à dor, aumentou a mobilidade articular e melhorou a autopercepção da saúde nesses pacientes (BO K, et al., 2015). O tratamento com laser em comparação a aplicação de Kinesiotaping (0\%), mostrou maior eficácia para os níveis gerais de saúde, com foco principal em depressão e ansiedade, entretanto, não existiu progresso quanto a dor noturna (MARQUES AP, et al., 2017). Um estudo realizado apenas com base em exercícios aeróbicos, onde os participantes eram monitorados por um frequencímetro, mostrou efetividade quanto a diminuição da variabilidade da frequência cardíaca, por meio da modulação cardiovagal. Além desses parâmetros, ocorreram melhoras significativas quanto a depressão e ansiedade nesses pacientes (MATHEUS SGJ, et al., 2016).

A maioria das interveções que proporcionaram melhoras, foram realizadas de forma individual. Todos os estudos citados tiveram participação do profissional de fisioterapia. As pesquisas trazem que a partir da diminuição dos sintomas físicos nos pacientes com fibromialgia, as complicações mentais, como os níveis de ansiedade, sofrem reduções. 


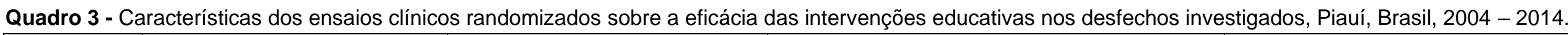

\begin{tabular}{|c|c|c|c|c|}
\hline Referência & $\begin{array}{l}\text { Delineamento/Número de } \\
\text { pacientes }\end{array}$ & Intervenções & Estratégia de acompanhamento & Desfechos e conclusões \\
\hline $\begin{array}{l}\text { Bravo C, et } \\
\text { al. (2018) }\end{array}$ & $\begin{array}{l}39 \text { participantes com } \\
\text { diagnóstico ACR } 1990 \text { para } \\
\text { FM, pelo menos } 6 \text { meses } \\
\text { antes do estudo e que } \\
\text { conseguissem ficar nas } \\
\text { posições deitado, sentado e } \\
\text { em pé sem assistência. } \\
\text { Idade: } 18-65 \text { anos } \\
\text { GC: } 20(53,50 \pm 9,19 \text { anos }) \\
\text { Gl: } 19(51,05 \pm 10,75 \text { anos })\end{array}$ & $\begin{array}{l}\text { GC: tratamento farmacológico } \\
\text { seguido de acordo com as } \\
\text { orientações do centro de } \\
\text { saúde primário } \\
\text { GI: movimentos BBAT (Basic } \\
\text { Body Awareness Therapy), } \\
\text { massagem hidropisia, e } 15 \\
\text { min para a partilha de } \\
\text { reflexões sobre as } \\
\text { experiências durante o } \\
\text { atendimento dentro do grupo. }\end{array}$ & $\begin{array}{l}\text { O BBAT foi realizado duas vezes por } \\
\text { semana durante } 5 \text { semanas, atendimentos } \\
\text { individuais de } 1 \text { hora cada. Os dois primeiros } \\
\text { atendimentos BBAT individualmente } \\
\text { consistiram em } 12 \text { movimentos. Durante os } \\
\text { atendimentos subsequentes, os pacientes } \\
\text { participaram de atendimentos em grupos de } \\
\text { BBAT, } 90 \text { min cada. Cada atendimento } \\
\text { incluiu movimentos BBAT, massagem } \\
\text { hidropisia, e } 15 \text { min para a partilha de } \\
\text { reflexões sobre suas experiências. }\end{array}$ & $\begin{array}{c}\text { Intervenção eficaz para a nossa } \\
\text { amostra de pessoas que sofrem } \\
\text { de FM em termos de dor, } \\
\text { qualidade de movimento, e } \\
\text { ansiedade, que foram } \\
\text { melhorados após a intervenção } \\
\text { BBAT. }\end{array}$ \\
\hline $\begin{array}{c}\text { Castel A, et } \\
\text { al. (2015) }\end{array}$ & $\begin{array}{c}130 \text { participantes com } \\
\text { diagnóstico ACR para FM, } \\
\text { sexo feminino. } \\
\text { ldade entre } 18 \text { e } 60 \text { anos } \\
\text { (idade média de } 49,2 \text { anos) } \\
\text { GC: } 61 \\
\text { Gl: } 69\end{array}$ & $\begin{array}{c}\text { GC: tratamento farmacológico } \\
\text { GI: tratamento farmacológico } \\
\text { seguido de terapia } \\
\text { comportamental cognitiva } \\
\text { (TCC) e fisioterapia. }\end{array}$ & $\begin{array}{l}\text { Todas as participantes passaram por um } \\
\text { estudo da doença. A terapia física enfatiza } \\
\text { tratamento para a capacidade aeróbia, } \\
\text { fortalecimento muscular e flexibilidade e } \\
\text { atendimentos de hidrocinesioterapia (em } \\
\text { piscina aquecida a } 30^{\circ} \text { ) e cinesioterapia } \\
\text { incorporada em um ginásio }\end{array}$ & $\begin{array}{l}\text { Os pacientes que se submeteram } \\
\text { ao tratamento multidisciplinar } \\
\text { para FM tiveram maior melhora } \\
\text { em comparação com os que } \\
\text { seguiram o tratamento } \\
\text { farmacológico convencional, com } \\
\text { relação a intensidade da dor, } \\
\text { catastrofização, distúrbios } \\
\text { psicológicos, funcionalidade, os } \\
\text { problemas do sono e a qualidade } \\
\text { de vida relacionada à saúde }\end{array}$ \\
\hline $\begin{array}{l}\text { Karlsson B, } \\
\text { et al. (2015) }\end{array}$ & $\begin{array}{c}48 \text { mulheres com diagnóstico } \\
\text { de FM } \\
\text { Idade entre } 18 \text { e } 64 \text { anos } \\
\text { Grupo } 2 \text { (GC): } 24 \\
\text { Grupo } 1(\mathrm{Gl}): 24\end{array}$ & $\begin{array}{c}\text { O grupo } 1 \text { recebeu o } \\
\text { tratamento com TCC, } \\
\text { enquanto o grupo } 2 \text { aguardava } \\
\text { em uma lista de espera (6 } \\
\text { meses). Quando a pirmeira } \\
\text { etapa foi concluida, o grupo } 2 \text {, } \\
\text { o grupo controle anterior, } \\
\text { recebeu o mesmo tratamento } \\
\text { de TCC que o grupo 1. Um } \\
\text { projeto de tratamento antes e } \\
\text { depois foi então realizado para } \\
\text { os dois grupos. }\end{array}$ & $\begin{array}{l}\text { A intervenção consistiu em } 20 \text { de TCC } \\
\text { durante um período de } 6 \text { meses. As } \\
\text { mulheres foram divididas em subgrupos de } \\
\text { tratamento com 5-7 mulheres em cada, } \\
\text { recebendo um atendimento de grupo por } \\
\text { semana, com a duração de } 3 \text { horas. Quando } \\
\text { os } 20 \text { atendimentos foram concluídos, três } \\
\text { atendimentos de reforço do grupo foram } \\
\text { dados durante os próximos seis meses, cada } \\
\text { um com duração de três horas. Cada } \\
\text { subgrupo teve o mesmo terapeuta. }\end{array}$ & $\begin{array}{l}\text { “Esgotamento vital" e } \\
\text { "comportamento de estresse" } \\
\text { foram reduzidas para ambos os } \\
\text { grupos. Concluiu-se que a TCC, } \\
\text { quando comparado a tratamento } \\
\text { de controle, pode até certo ponto, } \\
\text { reduzir a dor, humor negativo e } \\
\text { incapacidade no final do } \\
\text { tratamento e após o seguimento. } \\
\text { A dor foi avaliada com um } 6,3 \% \\
\text { de melhoria absoluta. }\end{array}$ \\
\hline
\end{tabular}

REAS/EJCH | Vol.Sup.n.51 | e3343 | DOI: https://doi.org/10.25248/reas.e3343.2020 Página 7 de 17 


\begin{tabular}{|c|c|c|c|c|}
\hline $\begin{array}{l}\text { Moretti EC, } \\
\text { et al. (2016) }\end{array}$ & $\begin{array}{l}15 \text { participantes com } \\
\text { diagnóstico de FM e parecer } \\
\text { cardiológico favorável à } \\
\text { prática de exercício. } \\
\text { Idade: } 18-60 \text { anos } \\
\text { GC: } 8 \text { (idade média } \\
44,75 \pm 13,44 \text { ) } \\
\text { Gl: } 7 \text { (idade média } \\
44,86 \pm 6,56 \text { ) }\end{array}$ & $\begin{array}{l}\text { GC: apenas alongamentos e } \\
\text { exercícios aeróbicos (EA). } \\
\text { GI: foi submetido a pompage, } \\
\text { alongamentos e EA. }\end{array}$ & $\begin{array}{l}\text { Durante } 12 \text { semanas, as voluntárias } \\
\text { passaram por dois atendimentos semanais. } \\
\text { As pompages foram aplicadas de forma } \\
\text { global (linfática, trapézios superiores, tronco, } \\
\text { lombar e quadríceps), } 5 \text { repetições com } \\
\text { manutenção de } 15 \text { segundos da tensão e } \\
\text { intervalos de } 10 \text { segundos). Foram } \\
\text { realizados alongamentos passivos de } \\
\text { isquiotibiais e de quadríceps femoral, e } \\
\text { ativos de cadeia posterior do tronco, cadeia } \\
\text { anterior do tronco e tríceps sural (duas } \\
\text { repetições de } 30 \text { segundos e } 10 \text { segundos } \\
\text { de intervalo). O EA era realizado em } \\
\text { bicicleta ergométrica, com intensidade de } 50- \\
60 \% \text { da frequência cardíaca máxima. } \\
\text { Sempre aumentando tempo e intensidade e } \\
\text { mantendo o tempo de intervalo ao decorrer } \\
\text { das semanas. }\end{array}$ & $\begin{array}{l}\text { A pompage associada a } \\
\text { exercícios de alongamento e EA } \\
\text { durante doze semanas para } \\
\text { tratamento da FM não apresentou } \\
\text { efeitos benéficos relevantes, uma } \\
\text { vez que melhorou apenas uma } \\
\text { das dimensões de dor avaliadas, } \\
\text { e nenhum efeito sobre a fadiga, } \\
\text { sono e ansiedade. }\end{array}$ \\
\hline $\begin{array}{l}\text { Wang C, et } \\
\text { al. (2018) }\end{array}$ & $\begin{array}{c}226 \text { participantes } \\
\text { diagnosticados com } \\
\text { fibromialgia (por meio da } \\
\text { ACR). } \\
\text { Idade: } 18 \text { e } 65 \text { anos } \\
\text { Atribuído ao exercício } \\
\text { aeróbico }(n=75) \\
\text { Atribuído a quatro grupos de } \\
\text { tai chi }(n=151)\end{array}$ & $\begin{array}{c}\text { Grupo } 1 \text { (tai chi): } 1 \\
\text { atendimento } \times 12 \text { semanas }(\mathrm{n} \\
=39) \\
\text { Grupo } 2 \text { (tai chi): } 2 \times \\
\text { atendimentos de } 12 \text { semanas } \\
(\mathrm{n}=37) \\
\text { Grupo } 3 \text { (tai chi): } 1 \\
\text { atendimento } \times 24 \text { semanas }(\mathrm{n} \\
=39) \\
\text { Grupo } 4 \text { (tai chi): } 2 \times \\
\text { atendimentos de } 24 \text { semanas } \\
\quad(\mathrm{n}=36) \\
\text { Grupo exercício aeróbico: } 2 \times \\
\text { atendimentos de } 24 \text { semanas } \\
(\mathrm{n}=75)\end{array}$ & $\begin{array}{c}\text { Os grupos de tai chi e o de exercícios } \\
\text { aeróbicos funcionaram simultaneamente. Os } \\
\text { cinco grupos receberam informação } \\
\text { educacional sobre a prática de atividades } \\
\text { físicas. Cada atendimento de tai chi durou } \\
60 \text { minutos, uma ou duas vezes por semana, } \\
\text { durante } 12 \text { semanas ou } 24 \text { semanas. Os } \\
\text { atendimentos de exercícios aeróbicos foram } \\
60 \text { minutos, duas vezes por semana, durante } \\
24 \text { semanas. }\end{array}$ & $\begin{array}{l}\text { Às } 24 \text { semanas os grupos de tai } \\
\text { chi melhoraram significativamente } \\
\text { mais do que o grupo de exercício } \\
\text { aeróbico. Foram encontradas } \\
\text { diferenças significativas em } \\
\text { melhorias na gravidade global } \\
\text { dos sintomas da fibromialgia, } \\
\text { bem como depressão, ansiedade, } \\
\text { auto eficácia, e componente } \\
\text { mental de qualidade relacionada } \\
\text { com a saúde da vida entre tai chi } \\
\text { e exercícios aeróbicos. }\end{array}$ \\
\hline
\end{tabular}

REAS/EJCH | Vol.Sup.n.51 | e3343 | DOI: https://doi.org/10.25248/reas.e3343.2020 Página 8 de 17 


\begin{tabular}{|c|c|c|c|c|}
\hline $\begin{array}{l}\text { Vitenet M, } \\
\text { et al. (2018) }\end{array}$ & $\begin{array}{l}28 \text { participantes com } \\
\text { fibromialgia (por meio da } \\
\text { ACR). } \\
\text { Idade: }>18 \text { anos } \\
\text { GC: } 14 \\
\text { Gl: } 14\end{array}$ & $\begin{array}{l}\text { Ambos os grupos foram } \\
\text { tratados com fisioterapia } \\
\text { durante a fase de teste. Ao } \\
\text { mesmo tempo, o grupo } \\
\text { experimental recebeu } \\
\text { atendimento de crioterapia }\end{array}$ & $\begin{array}{c}\text { Os participantes do grupo de crioterapia, } \\
\text { foram tratados a partir de um ciclo de } 10 \\
\text { visitas longo de um período de } 8 \text { dias. Eles } \\
\text { foram submetidos ao efeito de temperatura } \\
\text { extremamente baixa }\left(-110^{\circ} \mathrm{C} \text { ) em um }\right. \\
\text { cryoair, durante } 3 \text { min. A fim de proteger as } \\
\text { áreas do corpo mais sensíveis, todos os } \\
\text { pacientes que entraram estavam vestidos } \\
\text { com roupas de banho, máscara facial para } \\
\text { proteger o nariz e a boca, meias de algodão, } \\
\text { chinelos e luvas, orelha-protetor e sapatos } \\
\text { de madeira. }\end{array}$ & $\begin{array}{l}\text { Com alguns atendimentos de } \\
\text { crioterapia, ocorreram melhorias } \\
\text { rápidas (ou seja, dentro de } 1 \\
\text { mês) tanto nas dimensões físicas } \\
\text { e mentais de pacientes, } \\
\text { beneficiando a qualidade de vida } \\
\text { dos mesmos. }\end{array}$ \\
\hline $\begin{array}{l}\text { Ekici G, et } \\
\text { al. (2017) }\end{array}$ & $\begin{array}{l}43 \text { pacientes do sexo } \\
\text { feminino, com diagnóstica de } \\
\text { FM por meio da ACR. } \\
\text { Idade: }>25 \text { anos } \\
\text { Grupo } 1: 21 \\
\text { Grupo 2: } 22\end{array}$ & $\begin{array}{l}\text { Grupo 1: tratamento com } \\
\text { Pilates (PE) } \\
\text { Grupo 2: tratamento com } \\
\text { massagem tecidual (MT) }\end{array}$ & $\begin{array}{c}\text { Cada atendimento teve uma hora por } \\
\text { tratamento no grupo de PE. Este grupo de } \\
\text { tratamento consistiu em } 20 \text { minutos de } \\
\text { aquecimento e } \\
\text { esfriar (10 minutos antes e } 10 \text { minutos após } \\
\text { os exercícios) e } 40 \text { minutos de PE. O nível } \\
\text { de atividade foi aumentado gradualmente, de } \\
5 \text { repetições para 10. Com o tempo o número } \\
\text { de repetições foi aumentando. A MT foi } \\
\text { aplicado em posição sentada, sem suporte } \\
\text { nas costas. Um travesseiro foi colocado no } \\
\text { colo do participante para apoio do antebraço. } \\
\text { As costas do } \\
\text { os participantes estavam nuas e retas para a } \\
\text { tensão ideal do tecido conjuntivo. A MT foi } \\
\text { iniciada a partir da área lombo-sacral (seção } \\
\text { básica) e progrediu para a secções torácica, } \\
\text { escapular, interescapular e cérvico-occipital. } \\
\text { A MT demorou } 5 \text { a } 20 \text { minutos em cada } \\
\text { atendimento. }\end{array}$ & $\begin{array}{l}\text { Os resultados deste estudo } \\
\text { demonstraram que PE e MT } \\
\text { podem ser usados para fornecer } \\
\text { melhorias em mulheres com FM. } \\
\text { O tratamento com PE foi mais } \\
\text { eficaz do que a MT em termos do } \\
\text { limiar da ansiedade. Uma } \\
\text { possível explicação é que o } \\
\text { Pilates é composto de ativo, } \\
\text { simples e facilmente adaptável } \\
\text { exercícios. }\end{array}$ \\
\hline
\end{tabular}

REAS/EJCH | Vol.Sup.n.51 | e3343 | DOI: https://doi.org/10.25248/reas.e3343.2020 Página 9 de 17 


\begin{tabular}{|c|c|c|c|c|}
\hline $\begin{array}{l}\text { Bongi SM, } \\
\text { et al. (2016) }\end{array}$ & $\begin{array}{l}44 \text { pacientes diagnosticados } \\
\text { com fibromialgia por meio da } \\
\text { ACR. } \\
\text { GC: } 22 \\
\text { Gl: } 22\end{array}$ & $\begin{array}{l}\text { GC: participaram de um grupo } \\
\text { de ensino. } \\
\text { GI: participaram de } 3 \text { etapas. } \\
\text { Contemplando exercícios } \\
\text { respiratórios, posturais e } \\
\text { técnicas de Tai Ji Quan. }\end{array}$ & $\begin{array}{c}\text { No Gl, as etapas consistiram em, } 1^{\circ}(15 \\
\text { minutos): exercícios respiratórios, } \\
\text { concentração e manutenção postural com o } \\
\text { objetivo de aumentar a capacidade de } \\
\text { consciência corporal. } 2^{\circ} \text { ( } 15 \text { minutos): } \\
\text { exercícios permitindo reequilíbrio físico, } \\
\text { mental e emocional, melhorando posturas, } \\
\text { respiração e concentração, por meio de } \\
\text { movimentos com baixo impacto. } \\
3^{\circ} \text { (30 minutos): estudo e prática de uma } \\
\text { forma de Tai Ji Quan (TJQ) permitindo que o } \\
\text { paciente de realizar um movimento precisos. } \\
\text { TJQ estimula uma maior concentração e } \\
\text { coordenação nos movimentos. No GC, os } \\
\text { pacientes apenas participaram de um grupo } \\
\text { de ensino, a fim de dar informações sobre a } \\
\text { doença, gerenciamento de sintomas e da } \\
\text { capacidade de lidar com eles. }\end{array}$ & $\begin{array}{c}\text { Podemos supor que a melhora } \\
\text { sobre os sintomas de ansiedade } \\
\text { e sono é uma consequência da } \\
\text { eficácia do protocolo sobre a dor. } \\
\text { As técnicas de TJQ italiano em } \\
\text { pacientes FMS, reduzem a dor e } \\
\text { incapacidade, promovendo } \\
\text { melhora na qualidade de vida e } \\
\text { ansiedade. }\end{array}$ \\
\hline $\begin{array}{l}\text { Castro } \\
\text { Sánchez } \\
\text { AM, et al. } \\
(2018)\end{array}$ & $\begin{array}{l}64 \text { participantes } \\
\text { diagnosticados com SFM } \\
\text { Idade: } 18-60 \text { anos } \\
\text { Grupo 1: } 32 \\
\text { Grupo } 2: 32\end{array}$ & $\begin{array}{c}\text { Grupo 1: terapia com Dry } \\
\text { Needling } \\
\text { Grupo 2: terapia com liberação } \\
\text { miofascial }\end{array}$ & $\begin{array}{l}\text { Grupo 1: Todos os procedimentos de } \\
\text { agulhamento a seco, foram realizadas pelo } \\
\text { mesmo investigador, e a técnica foi } \\
\text { semelhante ao método de Hong. As } \\
\text { inserções da agulha em cada PGM foram } \\
\text { realizadas até se obter uma resposta de } \\
\text { contração muscular. A técnica foi aplicada } \\
\text { nos pares de músculos: occipitofrontais, } \\
\text { esternocleidomastóides, escaleno, trapézio, } \\
\text { supra-espinal, infra- espinal e multífidos. } \\
\text { Todos os pacientes receberam quatro } \\
\text { atendimentos (uma vez por semana). } \\
\text { Grupo 2: liberação miofascial profunda na } \\
\text { região temporal, liberação suboccipital, } \\
\text { compressão-descompressão da articulação } \\
\text { temporomandibular, liberação da região } \\
\text { peitoral, liberação diafragma (corrediça } \\
\text { transversal), e o plano do diafragma } \\
\text { transversal. }\end{array}$ & $\begin{array}{l}\text { Os benefícios obtidos por meio } \\
\text { de agulhamento seco parecem } \\
\text { ser maiores do que os obtidos } \\
\text { através da terapia miofascial no } \\
\text { curto prazo. A terapia de } \\
\text { agulhamento a seco de quatro } \\
\text { semanas reduziu } \\
\text { significativamente a sensibilidade } \\
\text { à pressão PGM, e melhoraram a } \\
\text { qualidade de vida, a qualidade do } \\
\text { sono, a ansiedade, a depressão, } \\
\text { o cansaço e a intensidade da dor, } \\
\text { em comparação com a terapia de } \\
\text { liberação miofascial. }\end{array}$ \\
\hline
\end{tabular}

REAS/EJCH | Vol.Sup.n.51 | e3343 | DOI: https://doi.org/10.25248/reas.e3343.2020 Página 10 de 17 


\begin{tabular}{|c|c|c|c|c|}
\hline $\begin{array}{l}\text { Da Silva } \\
\text { MM, et al. } \\
(2018)\end{array}$ & $\begin{array}{l}160 \text { participantes, do sexo } \\
\text { feminino com diagnóstico de } \\
\text { fibromialgia. } \\
\text { Idade: }>35 \text { anos } \\
\text { Grupo 1: Conjunto 1: } 20 \\
\text { Conjunto 2: } 20 \\
\text { Grupo 2: Conjunto 1: } 20 \\
\text { Conjunto 2: } 20 \\
\text { Grupo 3: Conjunto 1: } 20 \\
\text { Conjunto 2: } 20 \\
\text { Grupo 4: Conjunto 1: } 20 \\
\text { Conjunto 2: } 20\end{array}$ & $\begin{array}{l}\text { Os grupos foram divididos da } \\
\text { seguinte forma: Controle } \\
\text { (CON): doentes sob } \\
\text { tratamento farmacológico; } \\
\text { fototerapia (PHO): pacientes } \\
\text { submetidos à fototerapia; } \\
\text { treinamento físico (EXT): } \\
\text { pacientes submetidos ao } \\
\text { exercício físico e fototerapia } \\
\text { placebo; (Dispositivo de } \\
\text { fototerapia foi desligado como } \\
\text { um procedimento de } \\
\text { cegueira); fototerapia e } \\
\text { treinamento físico (PHO + } \\
\text { EXT). Todos os grupos foram } \\
\text { subdivididos em conjunto } 1 \text { e } \\
\text { conjunto } 2 . \text { No conjunto } 1 \text { foi } \\
\text { orientado para investigar o } \\
\text { efeito imediato de um único } \\
\text { atendimento de fototerapia. No } \\
\text { conjunto } 2 \text { foram realizados } \\
\text { para analisar o efeito a longo } \\
\text { prazo do } \\
\text { intervenções (10 semanas) na } \\
\text { condição de dor crônica e } \\
\text { outros sintomas de FM. } \\
\end{array}$ & $\begin{array}{l}\text { Fototerapia: As múltiplas fontes de luz (LBI e } \\
\text { LED) foram aplicadas em } 10 \text { pontos } \\
\text { sensíveis, em todos os pacientes (occipital, } \\
\text { do colo do útero (perto da C7), trapézio, } \\
\text { supra-espinal, epicôndilo lateral, glúteo, } \\
\text { sacro, trocanter maior e articulação } \\
\text { temporomandibular. Cada ponto foi irradiado } \\
\text { durante } 300 \mathrm{~s} \text {, e uma energia total } 39.3 \mathrm{~J} \text {. } \\
\text { Treinamento físico: alongamento ( } 30 \text { segs.) e } \\
\text { exercícios aeróbicos, duas vezes por } \\
\text { semana, durante } 10 \text { semanas. Alongamentos } \\
\text { estático ativo foram realizados para induzir } \\
\text { um leve desconforto nos seguintes grupos } \\
\text { musculares: bíceps, trapézio, grande dorsal } \\
\text { peitoral, paravertebrais, isquiotibiais e } \\
\text { quadríceps. Exercícios aeróbicos foram } \\
\text { realizados } 30 \text { min por atendimento. }\end{array}$ & $\begin{array}{c}\text { A terapia combinada provou ser } \\
\text { mais eficaz, principalmente para } \\
\text { ansiedade, depressão e fadiga. } \\
\text { Além disso, não foram } \\
\text { observadas diferenças } \\
\text { significativas após o tratamento } \\
\text { farmacológico em marcadores de } \\
\text { qualidade do sono. Verificou-se } \\
\text { que o funcionamento físico, } \\
\text { função emocional e a vitalidade } \\
\text { foram significativamente maiores } \\
\text { no grupo de PHO em relação ao } \\
\text { grupo CON. }\end{array}$ \\
\hline $\begin{array}{l}\text { Torres JR, } \\
\text { et al. (2015) }\end{array}$ & $\begin{array}{c}48 \text { participantes, } \\
\text { diagnosticados com } \\
\text { fibromialgia por meio da } \\
\text { ACR. } \\
\text { Idade: } 50-65 \text { anos } \\
\text { GC: } 24 \\
\text { Gl: } 24\end{array}$ & $\begin{array}{c}\text { GC: Intervenção educacional. } \\
\text { Gl: intervenção de mobilização } \\
\text { neurodinâmica. }\end{array}$ & $\begin{array}{l}\text { O GC recebeu um folheto que incluía } \\
\text { informações sobre cuidados posturais, } \\
\text { esportes, atividade física máxima sem dor, } \\
\text { aconselhamento comportamental sobre } \\
\text { medo do movimento, falsas crenças e um } \\
\text { estilo de vida ativo. } \\
\text { O GI recebeu intervenção de um programa } \\
\text { de mobilização neurodinâmica duas vezes } \\
\text { por semana durante } 8 \text { semanas } \\
\text { consecutivas. Cada atendimento durou } 60 \\
\text { minutos. }\end{array}$ & $\begin{array}{c}\text { Os resultados mostraram que a } \\
\text { mobilização neurodinâmica com } \\
\text { duração de } 8 \text { semanas } \\
\text { Proporcionou melhora } \\
\text { significativa na dor, ao estado de } \\
\text { saúde e } \\
\text { fadiga. O tratamento reduziu a } \\
\text { sensibilidade à dor, aumentou a } \\
\text { mobilidade articular e } \\
\text { melhorou a autopercepção da } \\
\text { saúde nesses pacientes. }\end{array}$ \\
\hline
\end{tabular}

REAS/EJCH | Vol.Sup.n.51 | e3343 | DOI: https://doi.org/10.25248/reas.e3343.2020 Página 11 de 17 


\begin{tabular}{|c|c|c|c|c|}
\hline $\begin{array}{l}\text { Silva HJA, } \\
\text { et al. (2019) }\end{array}$ & $\begin{array}{c}60 \text { participantes do sexo } \\
\text { feminino com diagnóstico de } \\
\text { fibromialgia (de acordo com } \\
\text { ACR). } \\
\text { Idade: } 18-60 \text { anos } \\
\text { Grupo } 1: 30 \\
\text { Grupo } 2: 30\end{array}$ & $\begin{array}{l}\text { Grupo 1: intervenção com } \\
\text { atendimentos de sofrologia. } \\
\text { Grupo 2: intervenção com } \\
\text { treinamento de resistência. }\end{array}$ & $\begin{array}{l}\text { Grupo 1: Um fisioterapeuta recitou algumas } \\
\text { frases tais como: "Fechar os olhos", "analisar } \\
\text { sua respiração e seu batimento cardíaco, a } \\
\text { fim de retarda-los", "imagine o seu corpo } \\
\text { relaxando progressivamente, músculo por } \\
\text { músculo "," "Sinto meu braço esquerdo muito } \\
\text { relaxado". Imagine um rio em uma floresta", } \\
\text { "sentir as águas deste rio passando entre } \\
\text { seus pés", "estas águas vão levando embora } \\
\text { toda a sua dor, o seu cansaço..." Ao entrar } \\
\text { em um estado de relaxamento, os pacientes } \\
\text { são convidados a pensar sobre a sua } \\
\text { doença e sua vida. } \\
\text { Grupo } 2: \text { um programa de treinamento } \\
\text { usando pesos para cálculo de uma repetição } \\
\text { máxima (1 RM), duas vezes por semana, } \\
\text { durante } 40 \text { minutos, por um período de } 12 \\
\text { semanas. }\end{array}$ & $\begin{array}{l}\text { A intervenção com sofrologia } \\
\text { mostrou resultados significativos, } \\
\text { diminuindo vários sintomas } \\
\text { desses pacientes, tanto físicos, } \\
\text { quanto mentais. O treinamento de } \\
\text { resistência mostrou melhoria da } \\
\text { força muscular, desemprenho no } \\
\text { teste de caminhada de } 6 \text { minutos } \\
\text { e capacidade funcional. }\end{array}$ \\
\hline $\begin{array}{c}\text { Moustafa } \\
\text { IM; Diab AA } \\
(2015)\end{array}$ & $\begin{array}{c}120 \text { participantes } \\
\text { diagnosticados com FM por } \\
\text { meio da ACR. } \\
\text { Idade: } 40-65 \text { anos } \\
\text { GC: } 60 \\
\text { Gl: } 60\end{array}$ & $\begin{array}{l}\text { Os pacientes em ambos os } \\
\text { grupos completaram um } \\
\text { programa multimodal de } 12 \\
\text { semanas consistindo de um } \\
\text { programa de educação, a } \\
\text { terapia comportamental } \\
\text { cognitiva, e um programa de } \\
\text { exercícios. Além disso, o } \\
\text { grupo de intervenção recebeu } \\
\text { terapia manipulativa cervical } \\
\text { superior. }\end{array}$ & $\begin{array}{l}\text { O programa de educação consistia em } 12 \\
\text { atendimentos, um por semana. Foram dadas } \\
\text { informações sobre os sintomas típicos, o } \\
\text { curso usual, condições médicas, potenciais } \\
\text { causas da doença, a influência de fatores } \\
\text { psicossociais na dor, farmacológicos e não- } \\
\text { farmacológicos tratamentos atuais, os } \\
\text { benefícios do exercício regular, e o típico } \\
\text { barreiras à mudança comportamental. A } \\
\text { terapia comportamental cognitiva teve } \\
\text { duração de } 12 \text { semanas. Consistiu em } \\
\text { combinação de diversos componentes que } \\
\text { incluem educacional, física, cognitiva, e } \\
\text { elementos comportamentais. A etapa física } \\
\text { contemplava respiraçãa diafragmática, } \\
\text { relaxamento progressivo, e visualização } \\
\text { guiada. A intervenção terapêutica } \\
\text { manipuladora consistiu em mobilização } \\
\text { articular. }\end{array}$ & $\begin{array}{l}\text { O tratamento com múltiplos } \\
\text { componentes, adicionado da } \\
\text { intervenção terapêutica } \\
\text { manipuladora é eficaz para } \\
\text { melhorar sintomas-chave da } \\
\text { FMS, incluindo dor, fadiga, } \\
\text { depressão, ansiedade e } \\
\text { qualidade de vida. }\end{array}$ \\
\hline
\end{tabular}

REAS/EJCH | Vol.Sup.n.51 | e3343 | DOI: https://doi.org/10.25248/reas.e3343.2020 Página 12 de 17 


\begin{tabular}{|c|c|c|c|c|}
\hline $\begin{array}{l}\text { VAYVAY } \\
\text { ES, et al. } \\
\text { (2016) }\end{array}$ & $\begin{array}{c}45 \text { participantes do sexo } \\
\text { feminino, diagnosticadas } \\
\text { com FM. } \\
\text { Idade: } \\
\text { Grupo 1: } 15 \\
\text { Grupo 2: } 15 \\
\text { Grupo 3: } 15\end{array}$ & $\begin{array}{c}\text { Grupo 1: intervenção com } \\
\text { laser } \\
\text { Grupo 2: intervenção placebo } \\
\text { laser } \\
\text { Grupo 3: intervenção com } \\
\text { kinesiotaping. }\end{array}$ & $\begin{array}{c}\text { Em todos os grupos foram administrados } 15 \\
\text { atendimentos, sendo } 5 \text { dias por semana } \\
\text { durante } 3 \text { semanas. } \\
\text { O laser foi aplicado durante } 3 \text { minutos para } \\
\text { cada um dos pontos dolorosos } \\
\text { predeterminadas, com o paciente em } \\
\text { decúbito ventral. A densidade de energia em } \\
\text { cada ponto foi definida para a exposição } \\
\text { radiante aproximadamente } 2 \mathrm{~J} / \mathrm{cm}^{2} \text {. A fim de } \\
\text { controlar o impacto do placebo, para os } \\
\text { indivíduos no grupo de estudo do laser } \\
\text { placebo, a aplicação foi realizada utilizando a } \\
\text { cabeça do laser sem dar energia. A } \\
\text { aplicação da kinesiotaping foi efetivada na } \\
\text { região torácica, nos músculos paravertebrais, } \\
\text { levantador da escapula e parte superior do } \\
\text { trapézio, com tensão de } 0 \% \text {. A kinesio foi } \\
\text { aplicada durante } 3 \text { semanas, e trocada de } 3 \\
\text { a } 5 \text { dias. }\end{array}$ & $\begin{array}{l}\text { De acordo com a EVA, não foi } \\
\text { estatisticamente significativa a } \\
\text { diminuição da dor noturna no } \\
\text { pós-tratamento. Enquanto } \\
\text { observa-se que nas três semanas } \\
\text { de Laser ocorreram melhoras no } \\
\text { nível geral de saúde, com foco na } \\
\text { depressão e ansiedade. Foi } \\
\text { notada maior funcionalidade. } \\
\text { A aplicação do laser, além disso } \\
\text { levou a diminuição dos níveis de } \\
\text { dor e o aumento da flexibilidade } \\
\text { do corpo. }\end{array}$ \\
\hline $\begin{array}{l}\text { Sañudo B, } \\
\text { et al. (2015) }\end{array}$ & $\begin{array}{l}32 \text { mulheres com diagnóstico } \\
\text { de FM. } \\
\text { Idade: } \\
\text { GC: } 16 \\
\text { Gl: } 16\end{array}$ & $\begin{array}{l}\text { GC: sem intervenção } \\
\text { GC: intervenção a partir de } \\
\text { exercícios aeróbicos }\end{array}$ & $\begin{array}{l}\text { O protocolo durou } 24 \text { semanas. Mulheres } \\
\text { alocadas para grupo de exercício aeróbico } \\
\text { realizaram duas atendimentos por semana } \\
\text { de } 45-60 \text { min de duração. Cada atendimento } \\
\text { incluiu } 10 \text { min de atividades de aquecimento } \\
\text { (movimentos fáceis e caminhada lenta), 15- } \\
20 \text { min de exercício de estado estacionário a } \\
60-65 \% \text { da frequência cardíaca máxima } \\
\text { prevista. A intensidade do exercício foi } \\
\text { monitorada por um sistema de telemetria da } \\
\text { frequência cardíaca. }\end{array}$ & $\begin{array}{l}\text { Os resultados deste estudo } \\
\text { indicaram que o treinamento de } \\
\text { exercício aeróbico pode melhorar } \\
\text { a variabilidade da frequência } \\
\text { cardíaca, principalmente pelo } \\
\text { aumento da modulação } \\
\text { cardiovagal. Melhoras } \\
\text { significativas também foram } \\
\text { encontrados em ansiedade e } \\
\text { depressão no grupo de exercício. }\end{array}$ \\
\hline
\end{tabular}

Legenda: FM: fibromialgia; EA: exercícios aeróbicos; GC: grupo controle; Gl: grupo de intervenção; ACR: Colégio Americano de Reumatologia; BBAT: comportamento básico de terapia comportamental; TCC: terapia comportamental cognitiva; MT: massagem tecidual; PE: Pilates; TJC: Tai Ji Quan; PGM: ponto gatilho; COM: controle; PHO: fototerapia; EXT: treinamento físico; PHO + EXT: fototerapia e exercício físico; RM: repetição máxima; EVA: Escala Visual Analógica. Fonte: Cunha SV, etal., 2020.

REAS/EJCH | Vol.Sup.n.51 | e3343 | DOI: https://doi.org/10.25248/reas.e3343.2020 Página 13 de 17 


\section{DISCUSSÃO}

Este estudo buscou evidencias quanto ao impacto de intervenções fisioterápicas na redução de complicações da fibromialgia, focando na ansiedade. A fisioterapia deve abandonar seu histórico de profissão ocupada apenas com a reabilitação dos pacientes (ANDREI PP, et al., 2016 e GEORGE SS, et al., 2008). Atualmente, o fisioterapeuta deve prezar pela saúde e aspectos relacionados à saúde dos pacientes, incentivando a prática de hábitos saudáveis, retirando o foco da doença e transferindo-o para a saúde dos indivíduos, protegendo-a, promovendo-a e, recuperando-a de forma integral (ANDREI PP, et al., 2016 e ROBIN H, et al., 2009).

Poucos estudos que analisaram as técnicas com relação às complicações foram encontrados, o que não necessariamente pode estar associado a não eficácia dessa intervenção, pois os estudos levantados nesta revisão sistemática, indicam relevância da fisioterapia perante aos níveis de ansiedade em pacientes com fibromialgia. Além disso, os métodos fitoterápicos, somados a educação desses pacientes, mostram progresso em várias outras complicações advindas da fibromialgia, como a dor, diminuída mobilidade e flexibilidade, cansaço, fadiga muscular, distúrbio do sono e depressão, promovendo assim, maior qualidade de vida para essas pessoas.

Estudos que não foram introduzidos nessa revisão, por conta dos fatores de exclusão, mostram concordância em seus resultados com algumas intervenções aqui citadas. No estudo de Marciele MZ, et al. (2018), foram analisadas técnicas de terapia manual onde apontaram melhoras na dor, ansiedade, depressão e contribuíram para uma melhor qualidade do sono.

Um estudo de Kyara MOMS, et al. (2012) aponta que imediatamente após a hidrocinesioterapia, observou-se melhora na ansiedade, sono, fadiga, rigidez e dor. Uma outra pesquisa também obteve resultados relevantes, onde os indivíduos tiveram declínio em sintomas da fibromialgia, como a ansiedade e fadiga através da hidrocinesioterapia (MATHEUS SGJ, et al., 2016).

A complexidade da síndrome fibromiálgica inclui fatores psicológicos, sociais e biológicos que requer uma abordagem biopsicossocial, preferencialmente de forma simultânea e não subsequente. $O$ tratamento não farmacológico frequentemente é associado com o tratamento farmacológico, incluindo a acupuntura, terapia cognitivo-comportamental, terapia corpo-mente, massagem, exercícios, hidroterapia, entre outras.

A acupuntura se mostra efetiva na redução da intensidade dolorosa em várias condições crônicas, incluindo a fibromialgia, além disso reduz a inflamação, libera opioides endógenos e diminui a ansiedade (JÚNIOR JOO e ALMEIDA MB, 2018).

Segundo Ziani MM, et al. (2017) a massagem relaxante aumenta a circulação sanguínea e de acordo com o toque e velocidade ocorre a liberação miofascial, desta forma, a pratica manual no tratamento da fibromialgia evidenciou efeitos positivos, e neste estudo foram obtidos resultados satisfatórios, melhorando a dor, a ansiedade e insônia nos pacientes com FM. As técnicas mais utilizadas foram drenagem linfática, liberação miofascial e alguns movimentos leves e profundos nos músculos incrementando reações fisiológicas, desativando pontos gatilhos ou tender point, ativando a circulação e como resultado proporcionando o relaxamento muscular.

Em relação a condicionamento físico, muitos pacientes que tem fibromialgia são fisicamente descondicionados, e, desta maneira, podem conseguir, através de programas de exercícios físicos que envolvem a ativação de condições analgésicas endógenas, incremento da sensação de bem-estar e melhora da qualidade de vida.

Em ensaios de exercício aeróbico e resistência, houve melhora do quesito dor, bem-estar físico e mental. O exercício em meio aquático foi considerado igualmente eficaz, tornando-se valioso para esses pacientes da mesma forma (JÚNIOR JOO e ALMEIDA MB, 2018).

Exercícios físicos têm sido indicados para aprimorar o bem-estar global em pacientes com fibromialgia, com objetivo principal de restabelecer os efeitos da falta de condicionamento físico, e melhorar os sintomas, especialmente a dor e a fadiga. Os exercícios de relaxamento corporal também podem surtir efeitos benéficos, mas não existe uma concordância em relação a isso, visto que geralmente são recomendados 
mais exercícios ativos do que passivos. Técnica como sofrologia, yoga, tai chi, acupuntura e música estão sendo citadas na literatura como alternativas complementares com efeitos promissores (SILVA HJA, et al., 2019).

Silva HJA, et al. (2019) expôs em seu estudo que a técnica de sofrologia é eficaz no tratamento de fibromialgia, pois apresentou benefícios nos quesitos saúde mental, aspectos sociais, dor e estado geral de saúde, e foi comparado com exercícios físicos resistidos realizados por aparelhos, duas vezes por semana, onde as cargas eram aumentadas progressivamente ao longo de doze semanas, e como resultado obtevese melhora na dor, força muscular, desempenho no Teste de Caminhada de 6 minutos e capacidade funcional.

Castro Sánchez AM, et al. (2018) em seu estudo utilizou a técnica de liberação miofascial em pacientes com fibromialgia e verificou melhora da ansiedade, qualidade do sono, dor e depressão tanto imediatamente após a intervenção, como em meses posteriores, neste estudo também foi utilizado a técnica de agulhamento a seco, perfazendo um total de 4 atendimentos por semana, onde apresentou seus benefícios mais a curto prazo, foi observado melhorias na qualidade de vida, qualidade do sono, na ansiedade, depressão, no cansaço e intensidade da dor.

Em um estudo comparativo entre exercícios de alongamento e exercícios aeróbicos em pacientes com fibromialgia, foi perceptível que existe uma eficácia superior dos exercícios aeróbicos nos sintomas de ansiedade em comparação aos exercícios de alongamento que influenciam diretamente na intensidade dor, reduzindo desta maneira, a quantidade de tender points, obtendo por consequência uma melhoria da qualidade do sono (MATSUTANI LA, et al., 2012).

Técnicas de cinesioterapia melhoraram a capacidade funcional, bem-estar, cansaço matinal, rigidez, ansiedade e depressão (BÁRBARA SMS, et al., 2017). A acupuntura promove uma analgesia benéfica somada à melhora do sono, ansiedade e depressão (FERNANDO RS, et al., 2017).

A corrente interferencial possui eficácia em relação à dor, incapacidade funcional, ansiedade, depressão e bem-estar global (ANDRADE GS, et al., 2018). Exercícios físicos trazem benefícios como, redução dar dor, níveis de ansiedade, depressão, melhoria da qualidade do sono e performance funcional (CRISTIANE PM, et al., 2017).

O tratamento fisioterapêutico pode intervir indiretamente na ansiedade. A dor crônica está comumente associada a transtornos psicoafetivos, como ansiedade e depressão, e transtornos do sono (ROBERT JG, et al., 2014). Distúrbios psicológicos estão fortemente ligados ao sono, e consequentemente, a falta dele tende a acarretar sintomas de ansiedade, estresse e contraturas musculares.

Recursos fisioterapêuticos, mesmo a curto prazo, provocam melhoria da dor, sono e capacidade funcional, em pacientes com fibromialgia (GOES SM, et al., 2017). Intervenções comportamentais para o sono mostraram impacto na redução da intensidade e incapacidade decorrente da dor e ansiedade (VIRGílIO R, et al., 2012).

A limitação para a formulação dessa revisão foi a escassez de estudos mais atuais e que de fato estudassem a efetividade e eficácia de intervenções que podem ser realizadas por fisioterapeutas, principalmente em relação à ansiedade, visto que os poucos estudos que retratam tal tratamento, relatam que a partir da eficácia quanto aos sintomas físicos, os níveis das complicações mentais sofrem declínio.

Outro ponto que levantamos como negativo, foi o não acompanhamento dos indivíduos que passaram pelos estudos por um tempo maior que um ano, para que ocorressem monitorizações mais fidedignas a respeito de suas qualidades de vida. Assim, não é possível saber se ocorreu evolução dos sintomas da FM.

Também pode ser analisado a partir desse estudo, que indivíduos diagnosticados com FM por menos tempo, obtiveram índice de melhora, mais efetivo do que aqueles que já obtiveram diagnóstico a mais de 30 anos, como por exemplo, a população idosa. Esse fato pode advir das próprias complicações mentais desses participantes. Mais estudos podem ser efetivados para conclusão dessa questão. Assim, reforça-se a importância desta revisão, cujos resultados retrataram a eficácia de distintas técnicas fisioterápicas, não somente aos níveis de ansiedade, mas para outras complicações sintomáticas da fibromialgia. 


\section{CONSIDERAÇÕES FINAIS}

Esta revisão identificou que a combinação de diferentes intervenções fornecidas individualmente ou em grupo a pacientes adultos com FM exerce influência na redução de complicações sintomatológicas. As intervenções eficazes envolveram atendimentos individuais de educação sobre a FM, o autocuidado e a importância da adesão ao tratamento na qualidade de vida e técnicas realizadas por fisioterapeutas ou equipe multidisciplinar (fisioterapeutas, médicos, psicólogos e educadores físicos). Essas ações melhoraram a níveis de dor, fadiga muscular, flexibilidade, mobilidade articular, distúrbio de sono, depressão e ansiedade. As intervenções mais efetivas recorreram a estratégias individuais, envolvendo encontros presenciais e contato telefônico nos estudos em que os pacientes deveriam efetivar atividades também em casa, ou realizar uso do fármaco, principalmente no caso dos grupos controle. Tais estratégias reduziram, principalmente, a frequência de dor, falta de sono, ansiedade e depressão dos pacientes.

\section{REFERÊNCIAS}

1. ADELAIDA M, et al. Improvement in clinical outcomes after dry needling versus myofascial release on pain pressure thresholds, quality of life, fatigue, pain intensity, quality of sleep, anxiety, and depression in patients with fibromyalgia syndrome. Disability and rehabilitation, 2018; 41(19): 1-12.

2. ANDRADE GS et al. Efeito da corrente interferêncial na funcionalidade em pacientes com fibromialgia. Semana de Pesquisa da Universidade Tiradentes - SEMPESq, 2018; (18).

3. ANDREI PP, et al. Análise do perfil biopsicossocial de participantes de um programa de educação em saúde específico para a fibromialgia. Conexão Ciência, 2016; 11(2): 92-102 apud George SS, et al. Utilização da Classificação Internacional de Funcionalidade, Incapacidade e Saúde na avaliação fisioterapêutica de indivíduos com problemas musculoesqueléticos nos membros inferiores e região lombar. Acta. Fisiátrica, 2008; 15(1): 24-30.

4. ANDREI PP, LUCIENE AF, NATANE MC, ANGÉLICA CSF, DÉBORA AR. Análise do perfil biopsicossocial de participantes de um programa de educação em saúde específico para a fibromialgia. Conexão Ciência, 2016; 11(2): 92-102 apud ROBIN H, et al. Validation of the International Classification of Functioning, Disability and Health Core Set for chronic widespread pain from the perspective of fibromyalgia patients. Arthritis research \& therapy, 2009; 11(3): 67.

5. BÁRBARA SMS, et al. Efeito dos tratamentos de hidroterapia, cinesioterapia e hidrocinesioterapia sobre qualidade do sono, capacidade funcional e qualidade de vida em pacientes fibromiálgicos. Life Style, 2017; 4(2): 35-53.

6. BO K, et al. Cognitive behaviour therapy in women with fibromyalgia: A randomized clinical trial. Scandinavian journal of pain, 2015; 9(1): 11-21.

7. BONGI SM, et al. Efficacy of rehabilitation with Tai Ji Quan in an Italian cohort of patients with Fibromyalgia Syndrome. Complementary therapies in clinical practice, 2016; 24: 109 - 115.

8. BORJA S, et al. Vagal modulation and symptomatology following a 6-month aerobic exercise program for women with fibromyalgia. Clin Exp Rheumatol, 2015; 33(1): 41-5.

9. BRAVO C, et al. Basic Body Awareness Therapy in patients suffering from fibromyalgia: a randomized clinical trial. Physiotherapy theory and practice, 2018; 35(10): 919-929.

10. CAMILA AO, et al. A eficácia da hidroterapia na redução da sintomatologia dos pacientes com fibromialgia. Revista Eletrônica Faculdade Montes Belos, 2016; 8(3): 1-179.

11. CASTEL $A$, et al. Body mass index and response to a multidisciplinary treatment of fibromyalgia. Rheumatology international, 2015; 35(2): 303-314.

12. CHAVES ECL, et al. Ansiedade e espiritualidade em estudantes universitários: um estudo transversal. Revista brasileira de enfermagem, 2015; 68(3): $504-509$.

13. CHENCHEN W, et al. Effect of tai chi versus aerobic exercise for fibromyalgia: comparative effectiveness randomized controlled trial. Bmj. 2018; 360:851.

14. CRISTIANE PM, et al. Efeito do exercício fisico em condições osteomioarticulares: revisão de literatura. Fisioterapia em Movimento, 2017; 22(4): 575-584.

15. EDUARDA CM, et al. Efeitos da pompage associada ao exercício aeróbico sobre dor, fadiga e qualidade do sono em mulheres com fibromialgia: um estudo piloto. Fisioterapia e Pesquisa, 2016; 23(3): 227-233.

16. EMRE SV, et al. The effect of Laser and taping on pain, functional status and quality of life in patients with fibromyalgia syndrome: A placebo-randomized controlled clinical trial. Journal of back and musculoskeletal rehabilitation, 2016; 29(1): 77-83.

17. FERNANDO RS, et al. A utilização da acupuntura no tratamento da fibromialgia relacionando com a qualidade de vida e a dosagem sérica de serotonina. Fisioterapia Brasil, 2017; 12(4): 298-302.

18. GAMZE E, et al. Effects of active/passive interventions on pain, anxiety, and quality of life in women with fibromyalgia: Randomized controlled pilot trial. Women \& health, 2017; 57(1): 88-107.

19. GOES SM, et al. Sono não-reparador e comorbidades associadas em mulheres com fibromialgia. Fisioterapia em Movimento, 2017; 22(3): 332-333. 
20. GONDIM SS, ALMEIDA MAPT. Os efeitos da massagem terapêutica manual em pacientes com a síndrome da fibromialgia. Revista multidisciplinar e de psicologia, 2018; 12(39): 336 - 354.

21. GUIMARÃES AMV, et al. Transtornos de ansiedade: um estudo de prevalência sobre as fobias específicas e a importância da ajuda psicológica. Caderno de Graduação - Ciências Biológicas e da Saúde - UNIT - ALAGOAS, 2015; 3(1): $115-128$.

22. IBRAHIM MM, ALIAA AD. The addition of upper cervical manipulative therapy in the treatment of patients with fibromyalgia: a randomized controlled trial. Rheumatology international, 2015; 35(7): 1163-1174.

23. JANET RT, et al. Results of an active neurodynamic mobilization program in patients with fibromyalgia syndrome: a randomized controlled trial. Archives of physical medicine and rehabilitation, 2015; 96(10): 1771-1778.

24. JÚNIOR JOO, ALMEIDA MB. O tratamento atual da fibromialgia. Brazilian Journal of Pain, 2018; 1(3): $255-262$.

25. KYARA MOMS, et al. Effect of hydrotherapy on quality of life, functional capacity and sleep quality in patients with fibromyalgia. Revista brasileira de reumatologia, 2012; 52(6): 851-857.

26. MARIANA MS, et al. Randomized, blinded, controlled trial on effectiveness of photobiomodulation therapy and exercise training in the fibromyalgia treatment. Lasers in medical Science, 2018; 33(2): 343-351.

27. MARQUES AP, et al. A prevalência de fibromialgia: atualização da revisão de literatura. Revista brasileira de reumatologia, $2017 ; 57(4): 356-363$.

28. MARTINS RCC, SILVEIRA NC. Eficácia da acupuntura para o tratamento da fibromialgia: uma revisão sistemática. Psicologia e saúde em debate, 2018; 4(1): 85 - 105.

29. MARTINS R, et al. Impacto da fibromialgia na qualidade de vida dos doentes. Servir, 2016; $59(3): 44-49$.

30. MATHEUS SGJ, et al. Hidrocinesioterapia na dor e na qualidade de vida em indivíduos portadores de fibromialgia. Rev Inspirar, 2016; 8(1): 29-33.

31. MATSUTANI LA, et al. Exercícios de alongamento muscular e aeróbico no tratamento da fibromialgia: estudo piloto. Fisioterapia em Movimento, 2012; 25(2): 411-418.

32. ROBERT JG, et al. Interdisciplinary chronic pain management: past, present, and future. American Psychologist, 2014; 69(2): 119, 2014.

33. ROBIN H, et al. Cinesioterapia versus hidrocinesioterapia no tratamento de mulheres fibromiálgicas: ensaio clínico randomizado. 6을 Congresso Internacional em Saúde, 2019; 11:67.

34. ROCHA MO, et al. Hidroterapia, pompage e alongamento no tratamento da fibromialgia-relato de caso. Fisioterapia em Movimento, 2017; 19(2).

35. RODRIGUES GF, et al. A relação entre fibromialgia e depressão. Trabalho de Conclusão de Curso - Faculdade Sant'ana, 2017; $18 \mathrm{p}$.

36. SILVA HJA, et al. Sophrology versus resistance training for treatment of women with fibromyalgia: A randomized controlled trial. Journal of bodywork and movement therapies, 2019; 23(2): 382-389.

37. VIRGíliO R, et al. $O$ efeito da hidroterapia na dor, qualidade de vida e funcionalidade em pacientes com fibromialgia. Universidade Fernando Pessoa - FCS, 2016; 1-17.

38. VITENET M, et al. Effect of whole body cryotherapy interventions on health-related quality of life in fibromyalgia patients: A randomized controlled trial. Complementary therapies in medicine, 2018; 36: 6-8.

39. WOLFE F. The status of fibromyalgia criteria. Arthritis \& rheumatology, 2015; 67(2): 330.

40. ZIANI MM, et al. Efeitos da terapia manual sobre a dor em mulheres com fibromialgia: uma revisão de literatura. Rev. Ciência e Saúde, 2017; 10(1): 48-55. 